\title{
Multiple Internet of Intelligences for Risk Analysis ${ }^{*}$
}

\author{
Chongfu Huang ${ }^{1,2}$ \\ 1. Academy of Disaster Reduction and Emergency Management, Beijing Normal University, Beijing 100875, China \\ 2. Beijing Cazl Technology Service Co., Ltd., Beijing 100088, China \\ E-mail: hchongfu@bnu.edu.cn
}

Received 24 April 2014

Accepted 4 June 2014

\begin{abstract}
In this study, we define a multiple Internet of intelligences (M-IOI) as the processing of homological information in layers. If agents in an M-IOI not only provide information in response to a question asked by a customer but also review information from other agents and summarize it, we refer to this as a summarizing M-IOI. The fuzzy mathematics method of normal diffusion is suggested to transform the summaries into fuzzy sets so that a satisfactory answer to the question is given. A summarizing M-IOI is used in a case study of typhoon dynamic risk in Wenzhou, China, where an insurance company wants to know whether the level of the risk will increase significantly. The effective knowledge in a summarizing M-IOI is measured to evaluate the quality of the answer. We also discuss the relation between IOIs and a global brain.
\end{abstract}

Keywords: Internet of Intelligences; Risk Analysis; Fuzzy Set; Normal Diffusion; Typhoon Dynamic Risk

\section{Introduction}

An Internet of intelligences (IOI) is a new website tool that collects and integrates messages, experience, knowledge, and judgment from intelligent agents to provide answers to questions from users (Huang, 2011). Unlike Google and Quora (Gannes, 2010), an IOI uses models to process the information from various agents to produce satisfactory answers.

A redesigned Google website could directly process "millions of different fact-seeking searches" and provide short answers at part of its results. "Google Questions and Answers" would allow users to collaboratively find satisfactory answers to their questions through the web. Quora considers the contributions it inspires a sort of "inverse blogging." If someone asks a question, it is because he or she wants an answer.

Google is a web search engine that allows users find other sites on the web based on keyword searches. Any answer suggested by Google must be supported by the sheer volume of web pages. Quora is a dialogue platform in which a user can ask a question on the Quora website and quickly receive answers from the network members who possess the relevant knowledge (Badilescu-Buga, 2013).

Neither Google nor Quora fully utilizes the pool of opinions on the Internet to answer questions. The main reason is that, although semantic analysis appears to be theoretically powerful on the web, it cannot process the missing structured data (Habernal and Konopik, 2013) to provide a satisfactory answer.

The concept of an IOI arose from online services for customers who have questions regarding some element of risk. There are always others who have experience, knowledge, and judgment concerning these questions (Huang, 2011). An IOI is an Internet-based service in which people employed by the service act as intelligent agents, and models are used to process the information provided by the agents.

\footnotetext{
* Project supported by the National Basic Research Program of China (973 Program) (No.2012CB955402), and partly supported by the Beijing Cazl Technology Service Co., Ltd..
} 
The first IOI was constructed to serve applicants to universities based on their entrance exam scores. The IOI collects and processes suggestions from college students and high school teachers. This system can help applicants choose among colleges and increase the probability of their being accepted to their first choice of schools, which is the greatest concern of their families. Another IOI has been used to survey the demand for typhoon insurance from the aquaculture industry in Wenzhou, China (Ai, 2014). The third IOI was designed to assist in writing proposals for grants. This IOI increases the chances of success for applicants facing a highly competitive environment and asymmetric information.

In practice, it is very difficult to build a complex mathematical model to process natural language information in an IOI that is both soft and flexible. To overcome this difficulty in this study, we propose a multiple IOI in which the agents not only provide information but also acquire knowledge by summarizing information.

This study is organized as follows. In Section 2, we define an IOI. In Section 3, we define a multiple IOI with layers. In Section 4, we suggest a multiple IOI to summarize information. Then, in Section 5 we employ the normal diffusion technique to integrate the summaries for risk analysis. Section 6 presents an application of a multiple IOI in analyzing typhoon dynamic risk. Section 7 presents a model to measure the effective knowledge in an IOI and the quality of service. Conclusions are provided in Section 8.

\section{Internet of Intelligences}

The concept of an Internet of intelligences (IOI) is based on the observation that everyone can act as an intelligent agent, having relatively more knowledge than others on some topic that may be used to help others to solve some problem through the Internet. For example, a tourist can answer questions regarding travel information (e.g., safety, discounts, or fraud) based on his/her experience. An IOI is intended to offer satisfying solutions for customers through human agents that apply their experience, knowledge and judgment to solve the customer's problems.

To define an IOI, we first define the following terms: agent, network and model.
An individual who can provide others with experience, knowledge, and judgment to solve problems is called an intelligent agent or simply an agent.

A computer system through which the agents can serve customers is called a network. The Internet is the most convenient network. In the remainder of the paper, unless stated otherwise, "network" refers to the Internet.

A scientific model is a representation of an object or a system. Any mathematical expression describing relationships among variables, any mathematical operation for processing information and any human brain paradigm for analyzing questions can be called a model. In the remainder of the paper, unless stated otherwise, "model" refers to a mathematical model or a human brain paradigm for processing information. For example, the linear regression method is a mathematical model. The set of rules used by an editor-in-chief of a journal is a human brain paradigm for processing the information in the comments from reviewers on papers submitted to the journal.

Slightly revising the definition given by Huang (Huang, 2011), we formally present a definition of an Internet of intelligences (IOI):

Definition 1. Let $A$ be a set of agents, let $N$ be a network used by $A$, and let $M$ be a model to process information provided by $A$. A triple $\langle A, N, M>$ is called an Internet of intelligences, denoted as $\Phi$.

An IOI is a network that provides knowledge products, where more than one intelligent agent is connected by a computer network and the agents' experiences, knowledge, judgment are collected and integrated by some model. If the model of an IOI is overly coarse, its product will be of low quality.

To evaluate an IOI $\Phi$, we suppose that there is a tool $T$ that can measure the intelligence level of $a_{i}$ in $A=\left\{a_{1}\right.$, $\left.a_{2}, \ldots, a_{n}\right\}$, i.e., $q_{i}=T\left(a_{i}\right)$. In addition, we assume that $Z$ can measure the intelligence level of $\Phi$, i.e., $Q=Z(\Phi)$. When $\mathrm{Q}>\max \left\{q_{1}, q_{2}, \ldots, q_{n}\right\}$, the IOI is called a positive IOI. Slightly revising the definition given by Huang (Huang, 2011), we have the following definition:

Definition 2. Let $\Phi=\angle A, N, M>$ be an Internet of intelligences with an intelligence level $Q$. Let the highest intelligence level of the individuals in $A$ be $q$. If $Q>q, \Phi$ is called a positive Internet of intelligences, denoted as $\phi \uparrow$. 
For example, let $A=\{$ John, Mary, Smith $\}$ be a team participating in a trivia quiz game. In this competition, all players must wait until the host reads a question, after which a light is turned on as a "ready" signal. The players press a button when they know the answer, and the first player to press their button wins the chance to respond. John, Mary and Smith use an IOI to compete, but other teams do not. That is, an IOI will compete with the other teams. In this case, the score is used to measure the intelligence level of a player. We suppose that the scores of John, Mary and Smith in a past competition are $q_{1}, q_{2}$, and $q_{3}$, respectively. The team's score is $q_{1}+q_{2}+q_{3}$. Without loss of generality, we assume $q_{1}>q_{2}, q_{3}$. When the team competes again using the IOI, the team's score is $S$. The intelligence level of the IOI is defined as $Q=S / 3$. If $Q>q_{1}$, the IOI is positive.

A simple IOI is illustrated in Fig. 1, and its topology is shown in Fig. 2.

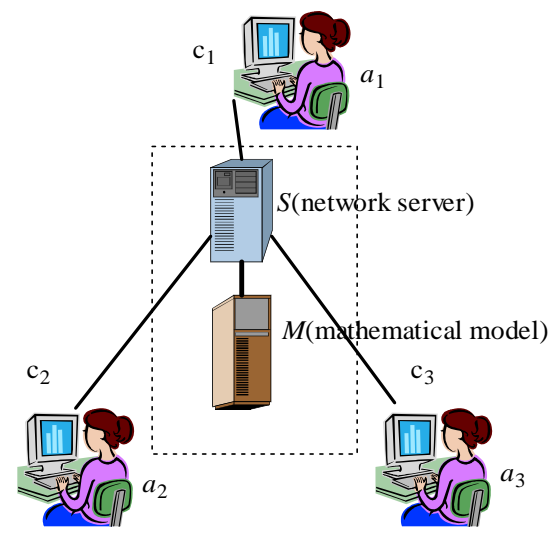

Fig. 1. A simple Internet of intelligences composed of a network server $S$, a mathematical model $M$, three computers $c_{1}$, $c_{2}$, and $c_{3}$ and three agents $a_{1}, a_{2}$, and $a_{3}$.

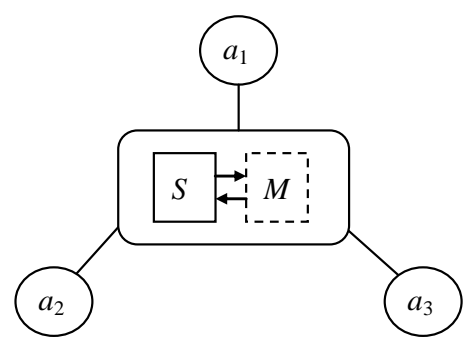

Fig. 2. Topology of the example Internet of intelligences composed of three nodes, where $S$ is the network server, $M$ is a mathematical model and $a_{1}, a_{2}$, and $a_{3}$ are agents.
The architecture of an IOI serving customers is illustrated in Fig. 3. The most important ingredient for constructing a positive IOI is to have a powerful model $M$ to extract and summarize knowledge from the information provided by the agents and then to construct knowledge products. Strictly speaking, it is the task of artificial intelligence to find $M$. Currently, IBM's "Watson", a supercomputer system capable of answering questions posed in natural language (Ferrucci et al., 2013), has the highest level of artificial intelligence. In a recent competition, Watson had access to 200 million pages of structured and unstructured content requiring four terabytes of disk storage. This content included the full text of Wikipedia, although the system was not connected to the Internet during the game. The algorithms used in "Watson" are very complex, and the system is supported by an extremely large database.

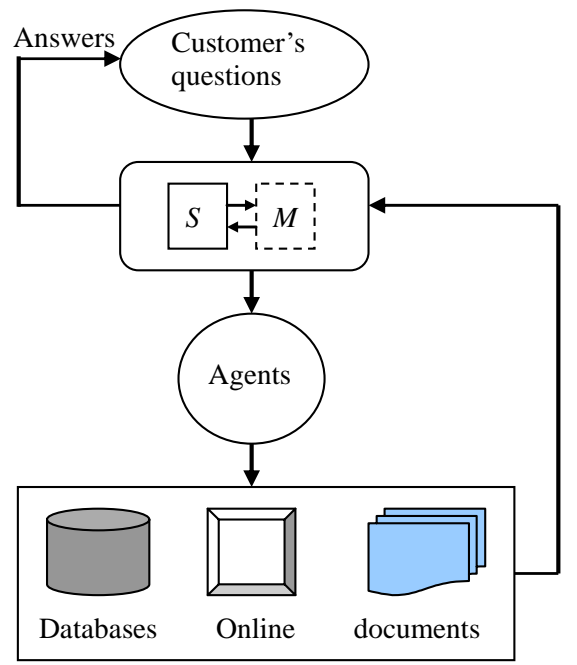

Fig. 3. An Internet of intelligences provides customers with answers to questions. Unlike other Q\&A systems, an IOI uses information from agents and applies models to process the information, where $S$ is a network server and $M$ is a mathematical model.

To alleviate the difficulty of discovering a complex model, in the next section we propose a multiple IOI to process information.

\section{Multiple Internet of Intelligences}

In practice, the information in an IOI might be heterogeneous, gathered from multiple sources such as natural language, databases and images. It is very 
difficult to construct a complex mathematical model to process such information. To alleviate this difficulty, the process is divided into several layers.

By analogy, files in the graphics editing software Photoshop can be composed of layers containing any manner of fills, strokes, images, fonts and miscellaneous objects to enable easy editing. It would be easier for an IOI to process homological information using the same approach. The homological information includes the experiences of agents, databases, monitoring systems, judgment, and reasoning that can be combined and modified using mathematical models. When the number of the layers in an IOI is greater than one, the IOI is called a multiple Internet of intelligences (M-IOI). Formally, we give the following two definitions:

Definition 3. Let $\Phi=<A, N, M>$ be an Internet of intelligences. A process for processing homological information in $\Phi$ is called a layer of $\Phi$.

Definition 4. Let $\Phi=<A, N, M>$ be an Internet of intelligences. If the number of layers of $\Phi$ is greater than one, $\Phi$ is called a multiple Internet of intelligences.

An M-IOI consisting of three layers is illustrated in Fig. 4. The information in each layer is homological; i.e., it is of the same type. Three separate models are used to process the individual layers. Lastly, a more complex

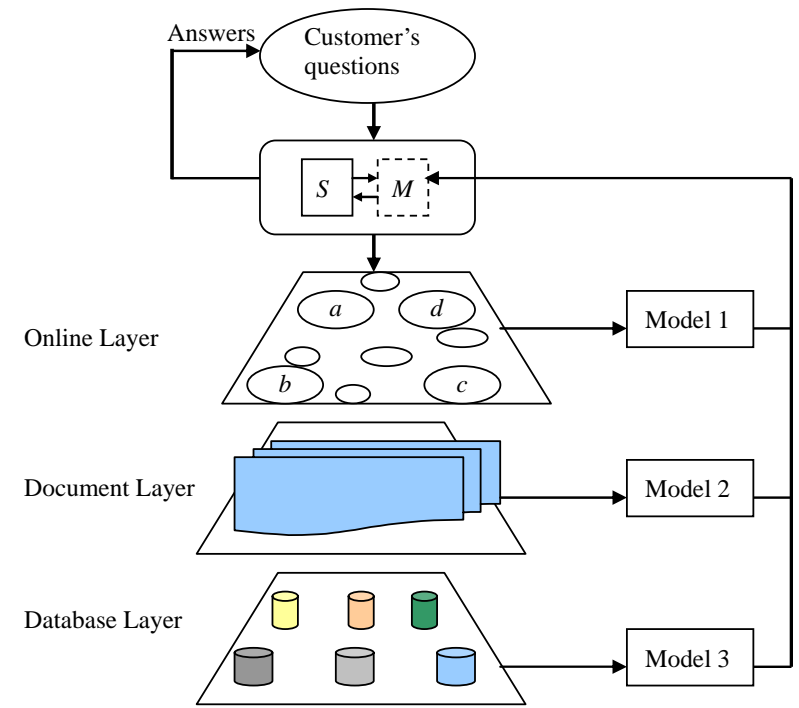

Fig. 4. A multiple Internet of intelligences consists of three layers to process online information, documents and databases separately, where $a, b, c, d, \ldots$ are agents. model is used to integrate the results from the models.

In an IOI, a model can be a scientific model, a human brain paradigm or a special tool. We use $M E$, $M O, B P, O T$ to denote mathematical expressions, mathematical operations, human brain paradigms and other tools, respectively. Then, a model $M$ in an IOI must be an element or a combination of elements of the set $\boldsymbol{M}$ in Eq. (1).

$$
\boldsymbol{M}=\{M E, M O, B P, O T\}
$$

The most difficult task is to process online information contained in natural language expressions; even the processing only summarizes the information. Because humans are better than any mathematical model at understanding and summarizing information in natural language expressions, we employ a human brain paradigm for summarizing information to create an MIOI.

\section{A Multiple Internet of Intelligences for Summarizing}

The simplest task for Model 1 in Fig. 4 is to summarize the information provided by agents responding to questions that are asked by a customer. We denote a question as $H$ and the response of agent $a$ as $e=a(H)$. If there are $n$ agents $a_{1}, a_{2}, \ldots, a_{n}$ responding to a question $H$, the IOI will have a set $E$ of $n$ responses, as shown in Eq. (2).

$$
E=\left\{e_{1}, e_{2}, \ldots, e_{n}\right\}
$$

The function of a "summarizing" model is to process $E$ and output a summary. If $e_{1}, e_{2}, \ldots, e_{n}$ are data, it is easy to find a statistical model to summarize them. However, if the responses are expressed in natural language, it is very difficult for a mathematical model to summarize the information. In this case, we invoke the agents to summarize. We do not know how an agent summarizes $E$, but we know that he/she is able to do so. The means for processing the natural language information is a human brain paradigm.

Without loss of generality, we assume that an agent $a$ summarizes $E$ and produces a summary $E_{a}$. For simplicity, the summary $E_{a_{i}}$ given by agent $a_{i}$ is written as $E_{i}$. In general, the summaries given by the agents will be different. If the summaries are expressed in natural language, it is difficult to find a mathematical model that will provide an integrated answer the customer's 
question. To obtain structured data, "summarizing" must be performed according to some rules.

A question arises if and only if there are uncertainties in a customer's mind. For example, if a businessman has to choose among four cities in a district to invest in a flower shop, his question is

$H$ : Which cite will give me the largest profit?

The profit depends on the drought risk and the number of customers (Huang and Inoue, 2007). The uncertainties in $H$ are "the drought risks in each of the four cities" and "the number of customers in each of the four cities”. In some sense, answering a question reduces or describes the uncertainties. This is the role of risk analysis.

Assuming that a question must be related to some uncertainties, an agent can summarize $e_{1}, e_{2}, \ldots, e_{n}$ by completing a form (e.g., a questionnaire) and thus reduce the uncertainties. Then, the sets $E_{1}, E_{2}, \ldots, E_{n}$ will be quantitative. That is, a set of responses $E$ to a question $H$ can be quantified using summaries. The summaries provide data samples that can be processed by a mathematical model to produce a satisfactory answer. Figure 5 shows the summarizing M-IOI.

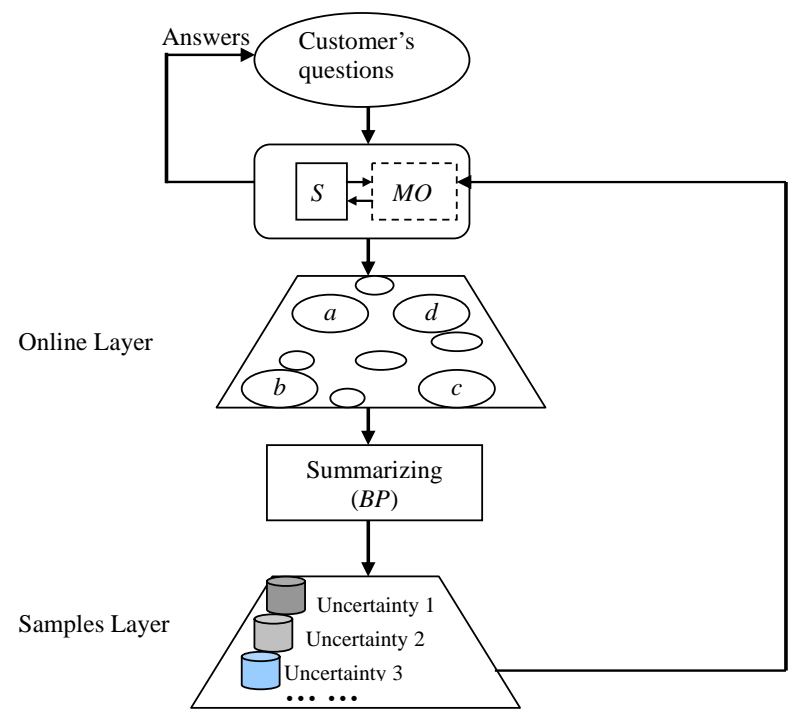

Fig. 5. A summarizing M-IOI consists of two layers. A human brain paradigm $(B P)$ titled "Summarizing" is used to process online information and output data samples. A mathematical operation $(M O)$ is used to process the samples. Here, $S$ is a network server, and $a, b, c, d, \ldots$ are agents.
In general, a customer purchases a service for the lowest possible price. The service is expected to be performed by some deadline. This expectation implies that the number of agents responding a question will be limited. When the size of a sample is small (below 30), we recommend the normal diffusion technique to process the samples.

\section{Normal Diffusion Technique for Risk Assessment}

The concept of information diffusion (Huang, 1997) was first introduced in function learning from a small sample of data (Huang and Moraga, 2004). The approximate reasoning of information diffusion was used to estimate probabilities and fuzzy relationships from scant, incomplete data for grassland wildfires (Liu et al., 2010). The interior-outer-set model, which is based on information diffusion theory, can be used to calculate a possibility-probability distribution from a small sample. This model provided better multi-valued results for flood risk management (Zou et al., 2012). Information diffusion theory was used to evaluate accident rates in dangerous chemical transportation and analyze the consequences of such accidents with GIS simulation technology (Zhang and Zhao, 2007). The use of information diffusion for fuzzy mathematics can be illustrated as follows (Huang, 2002).

Let $X=\left\{x_{i} \mid i=1,2, \cdots, m\right\}$ be a given sample and let $U=\{u\}$ be its universe. The function in Eq. (4) is called a normal diffusion function.

$$
\mu(x, u)=\exp \left[-\frac{(x-u)^{2}}{2 h^{2}}\right], \quad x \in X, u \in U .
$$

The diffusion coefficient $h$ can be calculated using Eq. (5) (Huang, 2012)

$$
h= \begin{cases}0.8146(b-a), & m=5 ; \\ 0.5690(b-a), & m=6 ; \\ 0.4560(b-a), & m=7 ; \\ 0.3860(b-a), & m=8 ; \\ 0.3362(b-a), & m=9 ; \\ 0.2986(b-a), & m=10 ; \\ 2.6851(b-a) /(m-1), & m \geq 11 .\end{cases}
$$

where $b=\max _{1 \leq i \leq m}\left\{x_{i}\right\}$ and $a=\min _{1 \leq i \leq m}\left\{x_{i}\right\}$. 
If $x_{i} \in X$ is a piece of information describing a risk, we can use the normal diffusion function to process $X$ for risk assessment. Specifically, when $x_{1}, x_{2}, \cdots, x_{m}$ are $m$ judgments, we can obtain a comprehensive judgment through the following steps:

Step 1: Using Eq. (5), calculate a diffusion coefficient $h$.

Step 2: Using Eq. (4) $\forall i, i \in\{1,2, \cdots, m\}$, transform a sample point $x_{i}$ into a fuzzy set with the membership function in Eq. (6)

$\mu_{x_{i}}(u)=\exp \left[-\frac{\left(x_{i}-u\right)^{2}}{2 h^{2}}\right], \quad u \in U$.

Step 3: Sum over all of the membership functions $\mu_{x_{1}}(u), \quad \mu_{x_{2}}(u), \cdots, \mu_{x_{m}}(u)$ to obtain the function

$$
f(u)=\sum_{i=1}^{m} \exp \left[-\frac{\left(x_{i}-u\right)^{2}}{2 h^{2}}\right], \quad u \in U
$$

Step 4: Find the absolute maximum $\left(u_{0}, f\left(u_{0}\right)\right)$ of $f(u)$ on $U$ using software such as Mathematica or MATLAB The critical point $u_{0}$ is the comprehensive judgment we seek.

The critical point $u_{0}$ is more robust than the simple average given in Eq. (8); i.e., when $m$ is small, a larger individual deviation will not significantly change the result.

$$
\bar{x}=\frac{1}{m}\left(x_{1}+x_{2}+\cdots+x_{m}\right)
$$

For example, assume the universe $U=[0,1]$, and let the judgments be $x_{1}=x_{2}=x_{3}=x_{4}=0.3, x_{5}=0.4$. We have

$$
\begin{gathered}
h=0.8146(0.4-0.3)=0.08146,2 h^{2}=0.01327 \\
f(u)=4 \exp \left[-\frac{(0.3-u)^{2}}{0.01327}\right]+\exp \left[-\frac{(0.4-u)^{2}}{0.01327}\right], \quad u \in U .
\end{gathered}
$$

The absolute maximum of $f(u)$ (see Fig. 6(a)) is at $(0.3124,4.515)$, so the critical point is $u_{0}=0.3124$. The simple average of $x_{1}, x_{2}, \ldots, x_{5}$ is

$$
\bar{x}=\frac{1}{5}(0.3+0.3+0.3+0.3+0.4)=0.32 \text {. }
$$

If the judgment of agent 5 differs more from the others, e.g., $x_{5}^{\prime}=0.8$, then the absolute maximum of
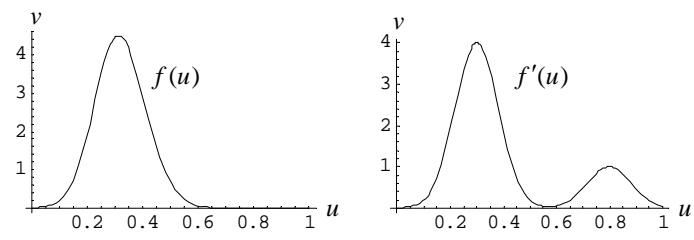
(a) $x_{1}=x_{2}=x_{3}=x_{4}=0.3$,
$x_{5}=0.4$
(b) $x_{1}=x_{2}=x_{3}=x_{4}=0.3$,

Fig. 6. The normal diffusion technique can robustly give a comprehensive judgment: a critical point. A larger individual deviation can substantially change a function, e.g., (a) versus (b), but the critical points for the absolute maximums, $(0.3124$, $4.515)$ and $(0.3,4)$, are not substantially different.

$f^{\prime}(u)$ (see Fig. 6(b)) will be at $\left(u_{0}^{\prime}, v^{\prime}\right)=(0.3,4)$. The simple average is $\bar{x}^{\prime}=0.4$. We have

$$
\left|u_{0}-u_{0}^{\prime}\right|=0.0124<0.08=\left|\bar{x}-\bar{x}^{\prime}\right| .
$$

This example demonstrates that the comprehensive judgment $u_{0}$ is more robust than the simple average $\bar{x}$.

In gymnastics competitions, the highest and lowest scores for an athlete are discarded to avoid biased judging. In risk analysis, however, any information is important, particularly when the sample size is small. In this case, the normal diffusion technique can provide a comprehensive judgment that is robust (no one individual can grossly affect a result with a significantly different input).

\section{A Case Study in Typhoon Dynamic Risk}

China is a country that has suffered devastation from numerous typhoons in its long history. On average, 7.2 typhoons affect China every year. Most of these typhoons occur on the southeastern coast of China. However, the typhoon risk is changing because of socio-economic development and population growth. One of the most important issues in planning for typhoon-related damage is to assess typhoon dynamic risk.

\subsection{Question and responses}

Suppose that a new insurance company is considering selling policies to clients in Wenzhou, a coastal city in Zhejiang province with nearly 60,000 residents. The company's profits will be sensitive to the risk of typhoon-related damage. If the level of risk is expected to increase in the next year, the company will not offer 
coverage to clients there, but if the risk is expected to decrease, the company will offer coverage.

In general, the company will conduct research or fund an external organization to study this problem. As a result, data on typhoons and resulting disasters will be collected and studied. In addition, reports published by the IPCC and the development plans for Wenzhou must be reviewed. This research will require a substantial amount of time and money. In contrast, a multiple IOI supported by climatologists, meteorologists and government offices could accomplish the task more quickly and at a lower cost.

Suppose that the company issues the following question: Our company provides insurance, and we are considering whether to sell policies in Wenzhou, where the risk of loss from typhoons is high. If the level of risk is likely to increase next year, possibly due to global warming, we will not sell policies there. If the level of risk is likely to decrease, possibly due to improvements in drainage systems, then we will sell policies there next year. We are unable to decide. Please advise us based on inputs from experienced professionals.

We assume that the company will pay $6000 \mathrm{RMB}$ to a service with an IOI, and each agent for the IOI will receive $1000 \mathrm{RMB}$ if he/she responds to the question with useful information. Furthermore, we assume that 5 agents respond; Table 1 lists the five responses.

\subsection{Quantitative summarizing}

When an IOI is employed to collect responses, we are confronted with the following problems:

(1) Some agents may maliciously provide false information in an attempt to discredit the online service.

(2) Agents may provide inconsistent information as a result of differing perspectives.

(3) The information may be incomplete because time and the budget are limited.

The IOI will filter false information and substantially inconsistent information. The information remaining is called the effective information.

We assume that the responses in Table 1 , which are denoted as $e_{1}, e_{2}, \ldots, e_{5}$, contain useful information. For example, $e_{1}=$ "Current information shows that global warming will not significantly affect typhoon activity".

Let Agent $i$, denoted as $a_{i}$, summarize all of the responses and give a summary $E_{i}$, as shown in Eq. (9). It should be noted that $\operatorname{Sum}_{i}$ will differ from $\mathrm{Sum}_{j}$ because of the different backgrounds of $a_{i}$ and $a_{j}$.

$$
E_{i}=\operatorname{Sum}_{i}\left\{e_{1}, e_{2}, \cdots, e_{5}\right\}
$$

To obtain structured data that will be analyzed using the normal diffusion technique, the agents are required to summarize the responses using Table 2, which is designed to reduce the five uncertainties in the question asked by the customer.

Table 1. Responses from 5 agents to the question of whether the risk of typhoon-related losses in Wenzhou is likely to increase.

\begin{tabular}{|c|c|c|}
\hline Agent & Discipline & Response \\
\hline Agent 1 & Climatologist & Current information shows that global warming will not significantly affect typhoon activity. \\
\hline Agent 2 & Meteorologist & $\begin{array}{l}\text { The records of typhoons that have affected Wenzhou show that the frequency of typhoons } \\
\text { has not significantly changed in recent years, but the intensity of the storms has increased. }\end{array}$ \\
\hline Agent 3 & $\begin{array}{l}\text { Civil Affairs } \\
\text { Bureau staff }\end{array}$ & $\begin{array}{l}\text { In Wenzhou, typhoon-related losses have increased approximately 5\% per year in recent } \\
\text { years. }\end{array}$ \\
\hline Agent 4 & $\begin{array}{l}\text { Statistics Bureau } \\
\text { staff in Wenzhou }\end{array}$ & $\begin{array}{l}\text { The annual growth of Wenzhou's gross domestic product (GDP) is approximately } 9 \% \text {. } \\
\text { Typhoon-related losses show a decreasing trend. }\end{array}$ \\
\hline Agent 5 & $\begin{array}{l}\text { Water Affairs } \\
\text { Bureau staff in } \\
\text { Wenzhou }\end{array}$ & $\begin{array}{l}\text { The government has been investing heavily to improve drainage facilities. In the next five } \\
\text { years, the standard to prevent a flood will be raised from a return period of } 30 \text { years to } 50 \\
\text { years. }\end{array}$ \\
\hline
\end{tabular}

Table 2. Questions for agents to quantitatively summarize responses.

\section{Will global warming affect the typhoon risk in Wenzhou?}

A. no information B. not at all C. slightly D. moderately E. significantly F. drastically

2. Will drainage facilities be improved?

A. no information B. not at all C. slightly $\quad$ D. moderately E. significantly $\quad$ F. drastically

3. In recent years, how did typhoon-related losses compare to GDP growth?
A. no information B. much lower

4. Is the typhoon risk decreasing?

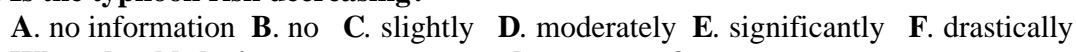

5. What should the insurance company do next year?

A. no information B. do not sell policies C. more data required D. further analysis required E. prepare to sell policies F. sell policies 
It is simple to transform a summary $E$ into a fivedimensional vector with the mapping function in Eq. (10).

$$
\left\{\begin{array}{l}
A \rightarrow-1 \\
B \rightarrow 0.00 \\
C \rightarrow 0.25 \\
D \rightarrow 0.50 \\
E \rightarrow 0.75 \\
F \rightarrow 1
\end{array}\right.
$$

Let $r_{i j}$ be the option of the ith agent to the $j$ th question. For example, Agent 1 chooses options C, E, C, $\mathrm{A}$, and $\mathrm{E}$, respectively, to the five questions. The summary $E_{1}$ is thus the vector: $\left(r_{11}, r_{12}, r_{13}, r_{14}, r_{15}\right)=(0.25$ $, 0.75,0.25,-1,0.75)$. In this case, the matrix in Eq. (11) shows all of the options of the agents for the five questions.

$R=\left(\begin{array}{lllll}r_{11} & r_{12} & r_{13} & r_{14} & r_{15} \\ r_{21} & r_{22} & r_{23} & r_{24} & r_{25} \\ r_{31} & r_{32} & r_{33} & r_{34} & r_{35} \\ r_{41} & r_{42} & r_{43} & r_{44} & r_{45} \\ r_{51} & r_{52} & r_{53} & r_{54} & r_{55}\end{array}\right)=\left(\begin{array}{ccccc}0.25 & 0.75 & 0.25 & -1 & 0.75 \\ -1 & 0.75 & 0.5 & 0.25 & 0.75 \\ 0.75 & 1 & -1 & 0.75 & 0.25 \\ 0.25 & 0.75 & 0.25 & 1 & 0.75 \\ 0 & 0.75 & 0.25 & 0.5 & 0.75\end{array}\right)$ (11)

\subsection{Processing summaries with the normal diffusion technique}

The elements in the first column of $R$ in Eq. (11) are the responses to "Will global warming affect the typhoon risk in Wenzhou? " A comprehensive judgment can be given by the normal diffusion technique with the sample

$$
X_{1}=\left\{r_{11}, r_{21}, r_{31}, r_{41}, r_{51}\right\}=\{0.25,-1,0.75,0.25,0\}
$$

where " $r_{21}=-1$ ” means in summary $E_{2}$, Agent 2 did not answer question 1 . The available set of answers to the

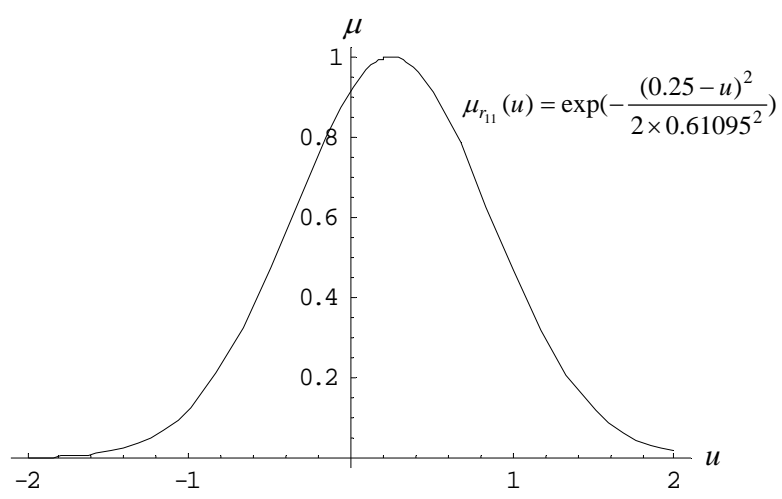

Fig. 7. A comment quantified as $r_{11}=0.25$ is transformed into a fuzzy set using the normal diffusion function. questions is

$$
X_{1}^{\prime}=\left\{r_{11}, r_{31}, r_{41}, r_{51}\right\}=\{0.25,0.75,0.25,0\}
$$

Using Eq. (5), we obtain the diffusion coefficient $h=0.8146(0.75-0)=0.61095$. Then, $r_{11}=$ 0.25 can be transformed into a fuzzy set as shown in Fig. 7.

Similarly, we transform other elements in $X_{1}^{\prime}$ into fuzzy sets. Summing the four membership functions, we have the function

$$
f(u)=2 e^{-1.33955(0.25-u)^{2}}+e^{-1.33955(0.75-u)^{2}}+e^{-1.33955 u^{2}}
$$

The absolute maximum of the function is at $(0.2925$, 3.642). Therefore, the comprehensive judgment on question 1 is $u_{1}=0.292506$. This value is closest to option C, namely, the answer is the following: Global warming will affect the typhoon risk in Wenzhou slightly.

Furthermore, the other columns lead to the following answers:

- Drainage facilities will be improved significantly.

- In recent years, typhoon-related losses were slightly lower than GDP growth.

- $\quad$ The risk typhoon will significantly decrease.

- $\quad$ Prepare to sell policies next year.

\section{Measuring the Effective Knowledge}

When we use a statistical model to analyze a relationship between two variables, the larger the sample is, the more accurate the result; and the better the statistical model is, the more reliable the result.

Similarly, the quality of service provided by an MIOI is determined by its effective knowledge and its model $\mathrm{M}$. The more effective knowledge is, the more credible the answer given by the IOI; and the better the model $\mathrm{M}$ is, the more reliable the answer.

In an M-IOI, the set $E$ of effective information shown in Eq. (2) will be provided by agents using their experience and judgment. For $E$, we assume $m$ agents provide $m$ summaries $E_{1}, E_{2}, \ldots, E_{m}$, which constitute a summary set $S$ shown in Eq. (12).

$$
S=\left\{E_{1}, E_{2}, \cdots, E_{m}\right\}
$$

When $m=n$, we suggest the following procedure to measure the effective knowledge in an M-IOI.

First, we assume that a customer will pay an amount of money $G$ for a service using an IOI, and any agent will receive compensation $g$ if he/she provides useful 
information, where $g<\mathrm{G}$. That is, $N=\operatorname{int}(G / g)$ is the expected number of agents employed by the service at one time, where "int" means to round to the next lower integer.

Second, we assume that $n$ agents provide useful information in answer to the customer's question. The ratio of $n$ to $N$, i.e., $n / N$, is called the basis of the effective knowledge, denoted as $b$. According to the previous assumption, the system will stop receiving information when $n$ is equal to $N$; i.e., the system receives information while $0 \leq b \leq 1$.

Any question asked by a customer must include some uncertainties. The task of the agents who provide information in an IOI is to reduce the uncertainties. The number of uncertainties in the question is denoted as $W$. If summary $E_{i}$ reduces $w$ uncertainties, $w$ is the dimension of $E_{i}$.

The average dimension of the summaries is $\bar{w}$. The ratio of $\bar{w}$ to $W$ is called the dimension of the effective knowledge, denoted as $d$; i.e., $d=\bar{w} / W$, and $0 \leq d \leq 1$

The effective knowledge from an M-IOI can be measured using Eq. (13), where $c$ is called the measure of effective knowledge. The closer $c$ is to 1 , the more credible the answer given by the M-IOI.

$$
c=b \times d=\frac{n}{N} \times \frac{\bar{w}}{W}=\frac{n}{\operatorname{int}(G / g)} \times \frac{\bar{w}}{W}
$$

We can use Eq. (13) to measure the effective knowledge of dynamic risk in the case of typhoons. We require the values of the variables in Equation (13), i.e., $n, G, g, \bar{w}$ and $W$. In our case, the company will pay $6000 \mathrm{RMB}$ for the service, and each agent will receive 1000 RMB. Therefore, $G=6000, g=1000,5$ agents provide effective information and $n=5$.

"Summarizing" is achieved by obtaining answers to the five questions in Table 2 and reduces the five uncertainties with the effective information given by the agents. Therefore, $W=5$. Option " $\mathrm{A}$ " means the agent did not reduce the corresponding uncertainty. An " $A$ " will reduce by one the dimension of $E_{i}$. For example, because there is an " $\mathrm{A}$ " in $E_{1}$, i.e., $r_{14}=-1$, the dimension of summary $E_{1}$ is $w_{1}=5-1=4$. Similarly, $w_{2}=4$, $w_{3}=4, w_{4}=5$ and $w_{5}=5$. The average dimension of the summaries $E_{1}, E_{2}, \ldots, E_{5}$ is

$$
\bar{w}=\frac{4+4+4+5+5}{5}=4.4
$$

The measure of effective knowledge is the following:

$$
c=\frac{n}{\operatorname{int}(Q / q)} \times \frac{\bar{w}}{W}=\frac{5}{\operatorname{int}(6000 / 1000)} \times \frac{4.4}{5}=0.733
$$

The value 0.733 means the final judgment (shown at the end of section 7) on typhoon risk in Wenzhou is not absolutely certain, but the judgment is nevertheless valuable for the insurance company when considering whether to insure clients in the region.

\section{Conclusions and Discussion}

The new online tool, the Internet of intelligences (IOI), answers questions from customers based on the experience, knowledge, and judgment of agents responding to the questions. Unlike other question-andanswer systems such as Google and Quora, an IOI applies models to process the information from multiple agents to arrive at satisfactory answers. An IOI is not a social networking site but a brain trust.

An IOI is defined as a triple $<A, N, M>$, denoted as $\Phi$, consisting of a set $A$ of agents, a network $N$ used by $A$, and a model $M$ to process information provided by $A$. When the intelligence level of $\Phi$ is greater than the highest intelligence level of the individuals in $A, \Phi$ is called a positive IOI, denoted as $\Phi \uparrow$.

In practice, the information in an IOI might be heterogeneous, obtained from multiple sources. We define a multiple Internet of intelligences (M-IOI) as the processing of homological information in layers, which allows the use of simpler models. In a summarizing MIOI, the agents are required to both provide and process the information. The small samples from summaries are optimally processed using the normal diffusion model to generate an integrated answer that is more robust.

An example of typhoon-related insurance risk shows that an M-IOI can predict for the customer, an insurance company, whether the level of risk will increase significantly. The methods reported in this study were applied to an IOI assessing the demand for typhoon insurance from the aquaculture industry in Wenzhou, China. Readers interested in this online system may refer to http://www.cazl.cn/ioi4ndra.

The Internet was based on the idea that there would be multiple independent networks of rather arbitrary design. As we now know, the Internet embodies a key underlying technical idea, namely that of open 
architecture networking. The predecessor to the Internet, the ARPANET, which was developed in the early 1970s by the U.S. Department of Defense (Tanenbaum, 1996), introduced several major innovations: email, the electronic text messages exchanged by users; a remote connection service for controlling a computer; and the file transfer protocol (FTP), which allows information to be sent from one computer to another in bulk. In the early 1990s, the World Wide Web (WWW) was created based on the Internet, a large and global TCP/IP network.

ARPANET provided the starting point for organizations to connect their computers. The web allowed the enormous growth of all types of "people-topeople" traffic. The evolution of the Internet is leading to a digital translation of the physical world, enabling the latter to be permanently connected. This is what is called the Internet of things. The next step is the Internet of intelligences. From the ARPANET to IOIs, the development of Internet routing is shown in Fig. 8.

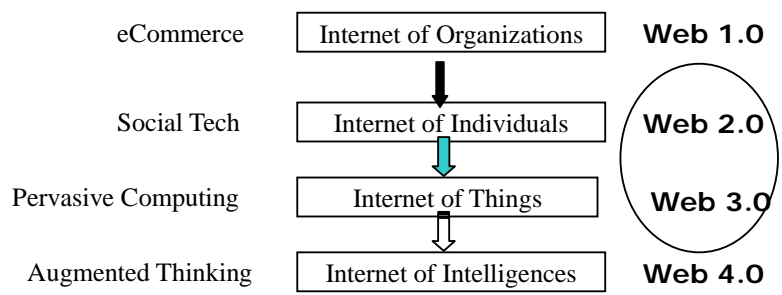

Fig. 8. The development of Internet routing: from Web 1.0 to Web 4.0. An Internet of intelligences is the future of the Internet. Currently, we are in the process of transition from Web 2.0 to Web 3.0.

As Internet speeds increase, it will be possible to share intelligences on a platform. The platform will be a part of the global brain, defined as a network that is an immensely complex, self-organizing system. A global brain not only processes information but can also play the role of a brain: making decisions, solving problems, learning new connections, and discovering new ideas. No individual, organization or machine is in control of this system; its knowledge and intelligence are distributed over all of its components (Heylighen, 2011). A global brain may be able to solve current and emerging global problems that have eluded more traditional approaches. At the same time, it will create new technological and social challenges that are as yet difficult to imagine. An IOI, as a part of a global brain, might offer a viable platform to solve extremely complex problems online through intelligent agents. A global brain supported by many IOIs is shown in Fig. 9 .

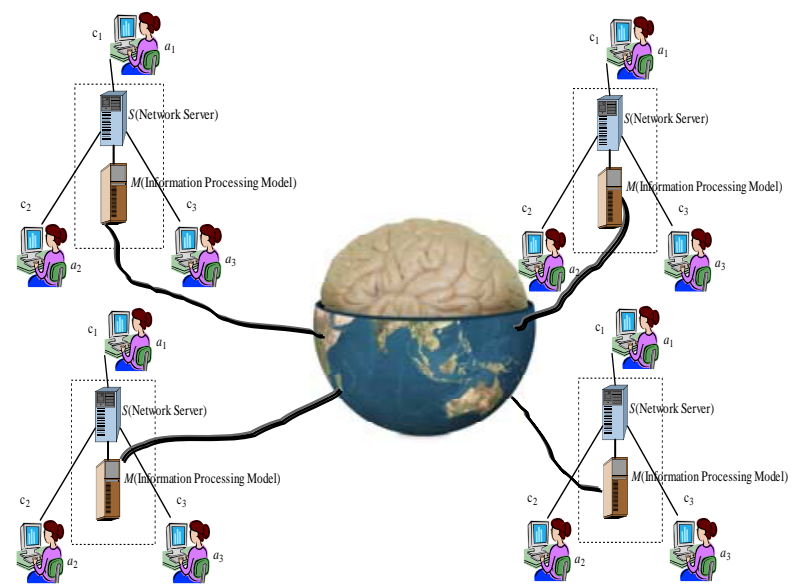

Fig. 9. In the future, a global brain would be supported by many Internets of intelligences to solve extremely complex problems online using intelligent agents, where each person plays a role as a brain cell.

There is no doubt that existing mathematical tools will not be sufficient to describe the future global brain. More powerful mathematical tools beyond differential calculus, probability theory and fractal theory will be required. Fuzzy logic (Zadeh, 1965, 1975, 1996) could be developed to support utilities to process flexible information expressed in natural language. In the future, a global brain could provide timely, effective and lowcost analysis of risks, making the world safer.

\section{References}

F. L. Ai, A Research on Building and Applying the Risk Analysis of Natural Disaster of Internet of Intelligences Service Platform, PhD Thesis, Beijing Normal University, Beijing, 2014.

E. Badilescu-Buga, Knowledge behaviour and social adoption of innovation, Information Processing and Management 49(4) (2013) 902-911.

D. Ferrucci, A. Levas, S. Bagchi, D. Gondek and E. Mueller, Watson: beyond Jeopardy! Artificial Intelligence (199200) (2013) 93-105.

L. Gannes, How quora is trying to build an ideal society (2010), http:// gigaom.com/2010/07/26/how-quora-istrying-to-build-an-ideal-society/.

I. Habernal, M. Konopik, SWSNL: semantic web search using natural language, Expert Systems with Applications 40(9) (2013) 3649-3664.

F. Heylighen, Conceptions of a Global Brain: an historical review, in Evolution: Cosmic, Biological, and Social, eds. 
L. E. Grinin, R. L. Carneiro, A. V. Korotayev and F. Spier (Uchitel Publishing, Volgograd, Russia, 2011) pp.274-289.

C. F. Huang, Principle of information diffusion, Fuzzy Sets and Systems 91(1) (1997) 69-90.

C. F. Huang, Information diffusion techniques and small sample problem, International Journal of Information Technology and Decision Making 1(2) (2002) 229-249.

C. F. Huang, Internet of intelligences in risk analysis for online services, Journal of Risk Analysis and Crisis Response 1(2) (2011) 110-117.

C. F. Huang, Risk Analysis and Management of Natural Disaster (Science Press, Beijing, 2012). (in Chinese)

C. F. Huang and H. Inoue, Soft risk maps of natural disasters and their applications to decision-making, Information Sciences 177(7) (2007) 1583-1592.

C. F. Huang and C. Moraga, A diffusion-neural-network for learning from small samples, International Journal of Approximate Reasoning 35(2) (2004) 137-161.

X. P. Liu, J. Q. Zhang, W. Y. Cai and Z. J. Tong, Information diffusion-based spatio-temporal risk analysis of grassland fire disaster in northern China, Knowledge-Based Systems 23(1) (2010) 53-60.

A. S. Tanenbaum, Computer Networks (Prentice Hall, Englewood, NJ, 1996).

L. A. Zadeh, Fuzzy sets, Information and Control 8(3) (1965) 338-353.

L. A. Zadeh, The concept of a linguistic variable and its application to approximate reasoning - I, Information Sciences 8(3) (1975) 199-249.

L. A. Zadeh, Fuzzy logic = computing with words, IEEE Trans. Fuzzy Systems 4(2) (1996) 103-111.

J. H. Zhang and L. J. Zhao, Risk analysis of dangerous chemicals transportation, Systems Engineering - Theory \& Practice 27(12) (2007) 117-122.

Q. Zou, J. Z. Zhou, C. Zhou, J. Guo, W. P. Deng, M. Q. Yang and L. Liao, Fuzzy risk analysis of flood disasters based on diffused-interior-outer-set model, Expert Systems with Applications 39(6) (2012) 6213-6220. 